\title{
ANÁLISE DA LOGÍSTICA INTERNACIONAL EMPREGADA PELO DEPARTAMENTO DE EXPORTAÇÃO DE UMA EMPRESA DE MÉDIO PORTE DA CIDADE DE VISTA ALEGRE DO ALTO - SP
}

\author{
ANALYSIS OF INTERNATIONAL LOGISTICS EMPLOYED BY THE DEPARTMENT \\ OF EXPORT OF A MEDIUM COMPANY OF THE CITY OF VISTA ALEGRE DO
}

$A L T O-S P$

Elvio Carlos da Costa - elvio.costa@ @fatectq.edu.br Faculdade de Tecnologia de Taquaritinga (FATEC) - SP - Brasil

DOI: 10.31510/infa.v15i1.321

\section{RESUMO}

O principal caminho para o crescimento da economia mundial é a ampliação do comércio internacional, uma vez que as nações dependem do intercâmbio comercial para atender as necessidades internas. $\mathrm{O}$ advento da globalização da economia, é imperativa a necessidade de redução de custos via adoção de sistema de integração da logística internacional. Nesse sentido, à medida que cresce o comércio internacional, as relações comerciais entre os países sofrem inúmeras transformações, dado o dinamismo que rege o processo econômico internacional. Exportar passou a ser uma questão de sobrevivência em um mundo cada vez mais integrado e globalizado. $\mathrm{O}$ objetivo deste trabalho consistiu em apresentar o processo da logística internacional empregada pelo departamento de exportação de uma empresa de médio porte da cidade de Vista Alegre do Alto - SP. Metodologicamente foi realizada uma pesquisa bibliográfica, seguida por uma pesquisa de campo, com abordagem qualitativa. Para a coleta de dados foi realizada uma entrevista estruturada com o gerente geral de uma empresa exportadora de médio porte da cidade de Vista Alegre do Alto - SP. Concluiu-se que a exportação é uma atividade praticável por qualquer tipo de empresa, de qualquer porte e setor. O empresário, no entanto, deve observar alguns aspectos importantes relacionados aos procedimentos iniciais de exportação: qual produto irá exportar, modais de transporte, custos logísticos, conhecer a legislação e cultura dos países que irá efetuar a exportação e estar atualizado em relação as taxas cambiais internacionais.

Palavras-chave: Comércio Internacional. Logística Internacional. Exportação.

\begin{abstract}
The main path for the growth of world economy is the amplification of international commerce, since the nations depend from commercial exchange to attend the internal necessities. With the advent of economic globalization, it is imperative the necessity for costs reduction through the adoption of an integration system of international logistic. This way, as the international commerce grows, the commercial relations among the countries suffer various transformations, given the dynamism that governs the international economic process. Exporting turned to be a matter of survivability in a world that gets more integrated and globalized. The objective of this work consisted to present the process of international
\end{abstract}


logistics used by the export department of a medium - sized company in the city of Vista Alegre do Alto - SP. Methodologically it was done a bibliographical research, followed by a camp study, where it was used a qualitative approach. For the data collect it was given a structured interview with the general manager of an exporting company of medium size in the city of Vista Alegre do Alto - SP. It can be concluded that exportation is an activity practiced by any kind of company, from any size or sector. The businessman, however, must notice some important aspects related to the initial procedures of exportation: which product to export, means of transportation, logistics costs, know the legislation and culture of countries he will perform the exportation and be up-to-date with the international exchange taxes.

Keywords: International Commerce. International Logistic. Exportation.

\section{INTRODUÇÃO}

A logística está se tornando uma área, das empresas em geral, que vem se destacando cada vez mais nos tempos atuais. Uma logística bem empregada poderá fazer com que a operação de uma determinada empresa, qualquer que seja, possa ser realizada da melhor maneira possível, realizando seus objetivos e reduzindo seus custos.

O conceito de logística integrada, "tem uma amplitude mais dinâmica e global, abrangendo não somente todas as tarefas pertinentes à gestão de materiais, como também a coordenação sobre os processos de manufatura, embalagens e manuseio, [...] distribuição e gerenciamento da informação". (RODRIGUES, 2002, p.138).

Para Lopez (2002) as empresas vêm buscando valorizar a especialização da logística, empregando-a em setores como os de compra de matéria-prima, logística de produção, logística de importação, logística de transporte, entre outros. Neste trabalho dar-se-á um maior destaque à logística empregada na operação de exportação, para que sejam analisados vários fatores que tendem a contribuir para uma operação de exportação bem ou mal sucedida.

É através da análise da logística empregada pela exportação que se pode obter a melhor forma de se exportar um determinado produto para um determinado país, escolhendose a forma e modalidade de transporte mais propício para a realização da mesma.

“A competitividade de uma empresa, [...] resulta de sua capacidade produtiva somada ao conhecimento e utilização adequada dos mecanismos para a oferta de seus produtos, à mão de seus consumidores, atendendo satisfatoriamente à trilogia preço, prazo e qualidade". (LOPEZ, 2000, p. 74). Verifica-se que a logística deve estar sempre levando em consideração vários aspectos pois, diversas vezes, a empresa deverá efetuar uma operação de exportação no menor tempo possível, independente dos custos. Da mesma forma, a empresa em questão 


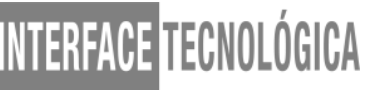

poderá buscar diminuir significativamente seus custos, não se importando com o seu transittime (tempo de trânsito de carga).

O tema escolhido deve-se à relevância da logística internacional utilizada pelas empresas contemporâneas, assim como o desejo do pesquisador em ampliar seus conhecimentos nesse assunto.

É notável a relevância dos estudos de uma logística bem empregada pelas empresas atuais. Com a situação cada vez mais difícil e com o mercado cada vez mais competitivo, as empresas exportadoras devem se preocupar com a forma em que são realizadas suas exportações, devendo empregar uma logística internacional confiável e segura.

Portanto, a situação problema levantada para a elaboração deste trabalho foi: "Análise da logística internacional. Assim, o objetivo deste trabalho foi apresentar o processo da logística internacional empregada pelo departamento de exportação de uma empresa de médio porte da cidade de Vista Alegre do Alto - SP.

O trabalho foi estruturado conforme a seguinte organização. Na introdução, foram demonstrados a relevância do tema pesquisado, o objetivo da pesquisa e a estrutura estabelecida do trabalho.

Na segunda seção foram abordados temas relacionados à logística internacional. Já na terceira seção, foram detalhados os procedimentos metodológicos, baseados em uma pesquisa bibliográfica, seguida por uma pesquisa de campo, com abordagem qualitativa. Para a coleta de dados foi realizada uma entrevista estruturada com o gerente geral de uma empresa exportadora de médio porte da cidade de Vista Alegre do Alto - SP.

Os resultados e discussões foram desenvolvidos na quarta seção, cuja finalidade foi apresentar o processo da logística internacional empregada pelo departamento de exportação de uma empresa de médio porte da cidade de Vista Alegre do Alto - SP.

E, por último, o trabalho foi concluído, onde foram expostas as considerações finais, baseadas na pesquisa bibliográfica e na entrevista estruturada aplicada.

\section{LOGÍSTICA INTERNACIONAL}

Tem-se observado uma aproximação cada vez maior dos mercados. Porém, é inaceitável quando se fala em internacionalização de mercados, não falar sobre logística. Esta é apontada como sendo um diferencial competitivo no escopo da gestão comercial. Por ser um 


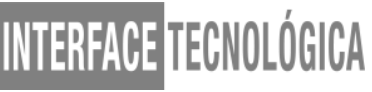

diferencial competitivo, a logística se encaixa perfeitamente dentro das necessidades das empresas de classe mundial.

Vários são os fatores que exigem das organizações uma rápida mudança e adequação às variáveis mercadológicas tais como: mudança de comportamento por parte dos consumidores, aumento da competitividade, redução do ciclo de vida do produto, opção por preço e não por marca e qualidade, entre outros. Com isso, é evidente que esta competitividade deixa de ser local, para ser global, gerando assim a necessidade de agilidade nas decisões estratégicas organizacionais (FIGUEIREDO; FLEURY; WANKE, 2007).

Para os autores, a logística é uma variável extremamente importante no atual contexto mundial e globalizado, com o mundo integrado e sem fronteiras. Isto se deve ao alto grau de competitividade que as empresas têm sido obrigadas a mostrar para que possam participar neste jogo internacional de comércio exterior cada vez mais disputado por empresas e países.

Para Ballou (2001) a logística está “[...] para agrupar conjuntamente as atividades relacionadas ao fluxo de produtos e serviços para administrá-las de forma coletiva é uma evolução natural do pensamento administrativo".

As demais atividades que fazem parte das relações comerciais internacionais são muito importantes. No entanto, a atividade de logística tem se destacado das demais. É ela quem está fazendo a diferença na competitividade das empresas. Isto tem ocorrido no plano mundial. Pode-se dizer que no Brasil o que vem ocasionando estas mudanças é a privatização de operações portuárias, privatização operacional da malha ferroviária, o renascimento da navegação de cabotagem (BALLOU, 2001).

Para Figueiredo, Fleury e Wanke (2007) internacionalmente, a abertura econômica mundial tem sido uma das principais responsáveis por este cenário. No atual cenário globalizado, o aumento da concorrência mundial tem impulsionado as empresas a atenderem satisfatoriamente aos seus clientes internacionais. Ligada a essa mudança comportamental dos mercados viu-se a necessidade de adaptação destes à medida que os relacionamentos entre empresas e clientes se desenvolvem e se afirmam.

Sendo assim, a logística vem em auxílio a estas organizações que procuram se destacar internacionalmente. Produtos semelhantes, várias empresas oferecem; o que irá diferenciar uma da outra será a forma, a rapidez e a eficiência com que estes chegarão aos seus destinos finais.

Historicamente, a palavra logística tem sua origem da palavra francesa loger, que quer dizer alojar. O exército francês usou este termo pela primeira vez, durante o século passado, 


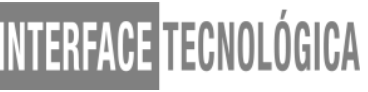

para definir as atividades realizadas pelos militares com relação à "arte de transportar, abastecer e alojar as tropas". Modernamente, o conceito evolui e a indústria passou a utilizálo para definir a arte de administrar o fluxo de informações e de materiais da fonte até ao consumidor final (MELLEN, 2012).

A logística "tem como objetivo [...] providenciar bens e serviços corretos, no lugar certo, no tempo exato e na condição desejada ao menor custo possível". Para oferecer tais serviços, a empresa exportadora tem a necessidade de conhecer bem o mercado para o qual vai exportar, além de conhecer a cultura deste (BALLOU, 2001).

De acordo com o Council of Logistics Management (Conselho de Gestão Logística) CLM, logística é:

“[...] o processo de planejamento, implementação e controle do fluxo eficiente e economicamente eficaz de matérias-primas, estoque em processo, produtos acabados e informações relativas desde o ponto de origem até o ponto de consumo, com o propósito de atender às exigências dos clientes”.

Desse modo, os componentes da logística, ou os elementos que a formam, são:

- processo de planejar, operar e controlar;

- fluxo e armazenagem (do ponto de origem ao ponto de destino);

- matéria prima;

- produtos em processo;

- informações;

- dinheiro.

Tais componentes devem ser de forma econômica, eficiente e efetiva além de satisfazer as necessidades e preferências do cliente.

Dentro da cadeia logística, cada elemento é cliente de seu fornecedor. É neste ponto em que há a necessidade de gestão da cadeia, a fim de se estabelecerem parcerias fidedignas e que estejam atendendo aos objetivos comuns dos demais componentes da cadeia, chegando-se assim ao seu objetivo primário: a satisfação plena do consumidor final. Por isso, dentro da cadeia, se faz necessário um rígido controle para que cada um dos elementos esteja de acordo ao modificarem seus processos e ao se adequarem a fim de atender às necessidades específicas almejadas (NOVAES, 2004).

Tendo estas premissas, para o autor, a logística moderna deve incorporar: 


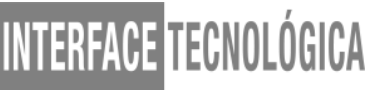

- prazos previamente acertados e cumpridos integralmente, ao longo de toda a cadeia de suprimento;

- integração efetiva e sistêmica entre todos os setores da empresa;

- integração efetiva e estreita (parcerias) com fornecedores e clientes;

- busca da otimização global, envolvendo a racionalização dos processos e a redução de custos em toda a cadeia de suprimento;

- satisfação plena do cliente, mantendo o nível de serviço preestabelecido e adequado.

\section{PROCEDIMENTOS METODOLÓGICOS}

Inicialmente, foi realizada uma pesquisa bibliográfica, com intuito de coletar todas as informações relacionadas a temática do trabalho, restringindo-se a definir objetivos e buscar mais informações sobre o tema, bem como publicações, artigos científicos e acadêmicos em torno do assunto em questão.

De acordo com Cervo e Bervian (2005) a pesquisa bibliográfica procura explicar um problema, a partir de referências teóricas publicadas em documentos, buscando-se conhecer e analisar as contribuições culturais ou científicas do passado, existentes sobre um determinado assunto, tema ou problema, cujos objetivos são: evitar duplicidade de pesquisa, determinar a contribuição para a base do conhecimento e obter fundamentação teórica para o desenvolvimento do projeto.

Posteriormente, para enriquecer o trabalho, foi realizada uma pesquisa de campo, onde foi utilizada a abordagem qualitativa. Para Michel (2005, p. 33) nesse tipo de pesquisa, não é possível comprovar a verdade, através de dados estatísticos ou de números, mas a partir de análises minuciosas, concisas e objetivas, em uma sequência lógica de ideias, o que permite que o pesquisador participe, compreenda e interprete os resultados.

Para a coleta de dados, o instrumento utilizado na pesquisa foi a entrevista. Segundo Lakatos e Marconi (2001, p. 195):

A entrevista é um encontro entre duas pessoas, a fim de que uma delas obtenha informações a respeito de determinado assunto, mediante uma conversação de natureza profissional. É um procedimento utilizado na investigação social, para a coleta de dados ou para ajudar no diagnóstico ou no tratamento de um problema social. 


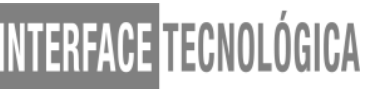

A entrevista padronizada ou estruturada, na qual o entrevistador segue um roteiro previamente estabelecido, foi aplicada ao Gerente do Departamento de Exportação e Logística Internacional de uma empresa de pequeno porte da cidade de Vista Alegre do Alto - SP.

A finalidade da entrevista foi coletar os dados referentes aos objetivos deste trabalho, que é analisar e descrever os procedimentos iniciais de exportação e apresentar o processo da logística internacional empregada pelo departamento de exportação da empresa em questão.

\section{RESULTADOS E DISCUSSÃO}

A entrevista estruturada foi aplicada com um profissional da área de Exportação e Logística Internacional da empresa case deste estudo, com 10 anos de experiência no ramo. O entrevistado foi escolhido por ter o perfil ideal, por ser Gerente Geral da empresa pesquisada e por ser graduado em Administração de Empresas e Direito, bem como pós graduado em Comércio Internacional. E os resultados foram expressos em categorias de análises, conforme segue:

\section{- Impacto da globalização e do avanço da tecnologia no comércio internacional}

De acordo como entrevistado, a globalização e a revolução tecnológica/organizacional são as duas principais forças motoras que estruturam as transformações no comércio internacional, e que definem algumas tendências marcantes no novo cenário de desenvolvimento econômico mundial.

Estas duas forças exercerão pressão, tanto em nível microeconômico por meio de estruturas produtivas e organizacionais, determinando quais empresas e organizações que sobreviverão com a intensificação da competição, quanto em nível macroeconômico e global, onde as mudanças tecnológicas e organizacionais estão relacionadas ao controle mais eficiente da economia mundial pelas transnacionais, através do controle sobre o crescimento do investimento em determinados países.

Portanto, a globalização ajudou as empresas a estarem visíveis ao mundo, e propiciou a uma empresa de médio porte do interior de São Paulo exportar produtos para os Estados Unidos da América (EUA) e China, porém como a empresa produz produtos primários, o avanço tecnológico não teve grandes impactos em sua produção. 


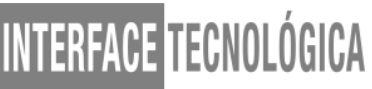

\section{- Conhecimento e regulamentação do comércio internacional}

Conforme o entrevistado, como os países não conseguem produzir todos os produtos de que necessitam, especializam-se nas atividades produtivas para as quais se encontram mais aptos, permutando os produtos entre si. Este comércio internacional ou comércio exterior submete os produtores internos a um maior grau de concorrência, reduzindo seu poder de mercado e, consequentemente, os consumidores internos compram produtos mais baratos, tanto dos produtores externos quanto dos produtores nacionais que devem manter seus preços competitivos.

Para o entrevistado, a regulamentação do comércio internacional está baseada principalmente em tratados internacionais entre os países, sob a batuta da Organização das Nações Unidas (ONU) e órgãos internacionais voltados ao comércio exterior, porém infelizmente a legislação brasileira trata muito superficialmente deste assunto.

- Vantagens da internacionalização e os obstáculos e desafios no processo de abertura do mercado global

Segundo o entrevistado, a internacionalização é uma das melhores formas de uma empresa alavancar seu crescimento. Boa parte das empresas que iniciam seu processo de exportação tem um crescimento considerável comparada às empresas concorrentes que não exportam. Além dos ganhos financeiros, em consequência do aumento das vendas, as vantagens da internacionalização são inúmeras, como: melhorar a competitividade no mercado interno, aumento dos lucros, possibilidade de contato com novas tecnologias, aumento do market share (participação de mercado), desoneração de alguns impostos, por exemplo: imposto sobre circulação de mercadorias e serviços (ICMS), imposto sobre produtos industrializados (IPI), programa de integração social (PIS), contribuição para o financiamento da seguridade social (COFINS), troca de experiência e ingresso de recursos em moedas estrangeiras. 


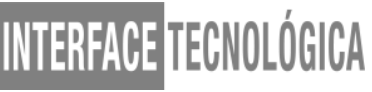

- Importância da logística internacional na competitividade da empresa em relação aos concorrentes

O aumento da concorrência mundial tem impulsionado as empresas a atenderem satisfatoriamente aos seus clientes internacionais. Com a mudança comportamental dos mercados, viu-se a necessidade de adaptação destes à medida que o relacionamento entre as empresas e clientes se desenvolvem e se firmam.

O entrevistado ressalta a importância da logística internacional, pois é a alma dos negócios internacionais, uma logística bem planejada faz toda a diferença. E acrescenta que a empresa a qual gerencia, não tem nenhum diferencial competitivo neste sentido, pois executa o básico.

\section{- Modal(is) de transporte(s) utilizado(s) pela empresa pesquisada}

A distribuição e transporte das mercadorias é de suma importância para o sucesso da empresa exportadora, pois deles depende parte importante da qualidade percebida pelo cliente internacional, isto é, o que ele sente ao comparar sua satisfação com suas expectativas. Confiabilidade de entrega é fruto do recebimento da mercadoria no prazo correto, com embalagem correta e sem danos causados pelo transporte.

A empresa pesquisada utiliza em suas transações internacionais o modal rodoviário até o porto de Santos, e de lá para o país de destino o modal marítimo, devido ao peso dos produtos que exportam.

\section{- Importância da logística integrada}

Entende-se logística integrada como sendo um canal integrado que vai da matéria prima ao fluxo reverso, tendo visão ampla e passando pelos departamentos de compra, produção, logística, pós venda etc. Porém a integração logística da empresa pesquisada é realizada de maneira parcial, ou seja, de forma separada, mantém parceria com duas diferentes empresas de transporte (rodoviário e marítimo) e várias áreas cuidam do processo de exportação. 


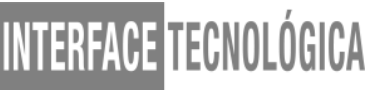

\section{- Custos logísticos}

Os custos logísticos estão relacionados com a logística de uma empresa, entre os quais se podem destacar os custos de armazenagem e transporte. Neste sentido, os custos logísticos da empresa pesquisada estão em transporte terrestre de container e transporte marítimo internacional. Para o entrevistado, entende-se que os custos nacionais estão competitivos e os custos internacionais poderiam ser mais baratos.

\section{- Processo da logística internacional empregado pelo departamento de exportação da empresa pesquisada}

O processo de logística utilizado é praticamente o transporte, onde as mercadorias são embarcadas em navios e a responsabilidade da empresa cessa com a entrega e com a chegada do navio no porto de destino, que seria a modalidade cost and freight (CFR), que é o que a empresa utiliza hoje, nesta modalidade o vendedor é o responsável pelo pagamento dos custos necessários para colocar a mercadoria a bordo do navio, pelo processo da exportação e também pelo pagamento do frete até o porto de destino designado.

\section{CONSIDERAÇÕES FINAIS}

A intensificação da globalização da economia mundial vem sendo acompanhada de profundas modificações econômicas e políticas nos últimos anos. A conjugação de novas tecnologias, com redução de tempo e custos, combinada com sistemas organizacionais, tem estimulado as empresas a fazerem alinhamentos na sua estrutura para tornarem-se competitivas. Entretanto, há praticamente um consenso entre os autores consultados sobre a importância e as mudanças significativas no comércio e logística internacional.

O comércio internacional vem mudando ao longo dos tempos junto com as modernizações das práticas de comércio e o desenvolvimento de novas tecnologias. O avanço industrial, dos transportes, a globalização, o surgimento das corporações transnacionais tiveram grande impacto no incremento deste comércio.

Nesta era de crescente interdependência mundial, o comércio internacional é extremamente importante para o crescimento econômico de todos os países. Neste sentido, 


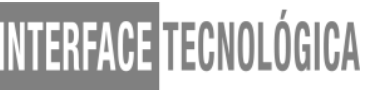

este tipo de comércio não é somente do domínio das grandes organizações, de fato, as pequenas e médias empresas também estão inseridas no mercado global.

Com base no objetivo estabelecido para o presente trabalho, observou-se que a tarefa mais desafiadora para uma empresa que desejar exportar é ter conhecimentos plenos da legislação e da cultura do país para onde pretende exportar seus produtos, bem como ter conhecimento holístico sobre os procedimentos iniciais de exportação, como: taxas cambiais, modais de transporte, marketing e parcerias internacionais e custos logísticos.

É fundamental que os membros da organização participem das atividades de planejamento da distribuição logística de forma integrada, pois propicia à empresa uma maior competitividade global.

Conforme a pesquisa realizada, pode-se observar que a competitividade tem exigido que as empresas desenvolvam vantagens competitivas em relação a seus concorrentes que envolvem tempo, custo e nível de serviços. O gerenciamento logístico internacional estratégico surge como uma ferramenta poderosa, com o objetivo de oferecer aos gestores parâmetros de avaliação do desempenho compatível com os objetivos da empresa.

Os custos logísticos podem ser gerenciados de forma a minimizar os gastos e maximizar a qualidade e os resultados nos negócios, consequentemente, agregar valor aos sócios ou acionistas das empresas.

Verificou-se no trabalho de pesquisa realizado, que os modais mais utilizados são os rodoviários e marítimos, por questões de preço e peso, respectivamente. E que a maior dificuldade encontrada pelas empresas brasileiras na inserção e permanência no comércio internacional é o problema com a flutuação do câmbio.

A gestão de todo o processo logístico é complexa, envolve vários fornecedores, diversos locais de entrega e contratos não padronizados. Portanto, isso faz com que muitas empresas exportadoras assumam uma postura reativa no que diz respeito à cotação, avaliação, seleção e gestão das contratações de prestadores de serviços de transporte logísticos.

Por fim, conclui-se que a área do comércio e logística internacional tem crescido de forma ordenada, possibilitando que as empresas terceirizem suas atividades e se dediquem ao seu foco de negócio, pois desta forma se tornarão mais competitivas em um mercado altamente competitivo e globalizado e, sobretudo, elevando níveis de serviços.

Porém, mesmo vivendo um crescimento acelerado, o comércio internacional e o setor logístico ainda necessita de muitos investimentos, tanto do setor público quanto do privado, a fim de minimizar problemas e trazer desenvolvimento para o setor e para a economia do país. 


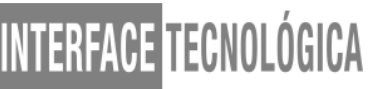

\section{REFERÊNCIAS}

BALLOU, R. H. Gerenciando a cadeia de suprimentos: planejamento, organização e logística empresarial. Porto Alegre: Bookman, 2001.

CERVO, A. L.; BERVIAN, Pedro A. Metodologia científica. 5. ed. São Paulo: Person Prentice Hall, 2005.

FIGUEIREDO, K. F.; FLEURY, P. F.; WANKE, P. Logística empresarial: a perspectiva brasileira. São Paulo: Atlas, 2007.

LAKATOS, E. M.; MARCONI, M. A. Fundamentos de metodologia científica. 4. ed. São Paulo: Atlas, 2001.

LOPEZ, J. M. C. Os custos logísticos do comércio exterior brasileiro. São Paulo: Aduaneiras, 2000.

LOPEZ, J. M. C.; GAMA, M. Comércio exterior competitivo. São Paulo: Aduaneiras, 2002.

MELLEN, J. A origem logística e sua história. Disponível em: <http://www.guiadelog.com.br>. Acesso em: 06 jan. 2018.

MICHEL, M. H. Metodologia e pesquisa científica em ciências sociais: um guia prático para acompanhamento da disciplina e elaboração de trabalhos monográficos. São Paulo: Atlas, 2005.

NOVAES, A.G. Logística e gerenciamento da cadeia de distribuição. Rio de Janeiro: Elsevier, 2004.

RODRIGUES, P. R. A. Introdução aos sistemas de transporte no Brasil e à logística internacional. 2. ed. São Paulo: Aduaneiras, 2002. 